\title{
Virtual voice clinics in the COVID-19 era: have they been helpful?
}

\author{
Carolina Watters $^{1} \mathbb{D} \cdot$ Benjamin Miller ${ }^{2} \cdot$ Mairead Kelly $^{1} \cdot$ Victoria Burnay $^{3} \cdot$ Yakubu Karagama $^{4} \cdot$ Elfy Chevretton $^{4}$
}

Received: 29 November 2020 / Accepted: 21 January 2021 / Published online: 24 March 2021

(c) Crown 2021

\begin{abstract}
Purpose In response to the coronavirus pandemic, a tertiary combined Laryngology-Speech Therapy voice clinic was converted to a wholly virtual clinic, with consultations carried out via telephone or video. The aim of our mixed method study was to assess (a) how effective are virtual clinics vs face-to-face clinics in progressing patients' care and (b) what is patient satisfaction with virtual consultation methods.

Methods Analysis of clinic data from patient databases for both virtual and face-to-face clinics was carried out. A patient satisfaction survey was carried out by 75 of the patients who had attended virtual clinics.

Results There was statistically a significant difference $(p$ value $<0.01)$ in the proportion of patients prescribed medical therapy, referred for Speech and Language Therapy (SALT) or listed for surgery in the virtual clinic by comparison to the face-to-face clinic. 75 patients completed the questionnaire. $98 \%$ of patients were satisfied overall with the virtual method of consultation. $84 \%$ believed they would still benefit from face-to-face review. $83 \%$ would like the option of a virtual type of clinic in the future.

Conclusion Our data clearly demonstrates that face-to-face clinics are superior to virtual clinics, with almost no patients progressed to surgery in virtual consultations. Despite this, virtual methods are still valuable, and many patients have meaningful progression of care. In current circumstances, patients have very high satisfaction with virtual consultations and certain groups have been identified as particularly benefiting. Going forward, an ideal clinic may be a hybrid of face-to-face and virtual appointments as clinically indicated.
\end{abstract}

Keywords COVID-19 $\cdot$ Voice $\cdot$ Laryngology $\cdot$ Clinics $\cdot$ Virtual $\cdot$ ENT

\section{Background}

The COVID-19 pandemic has necessitated clinicians from all medical and surgical specialties to practice in new and innovative ways. In March 2020, ENT UK guidance advised that wherever possible ENT outpatient clinics should be kept to a safe minimum, with an emphasis on the provision

Carolina Watters

carolinawatters@hotmail.co.uk

1 Core Surgical Trainee, ENT Department, Guy's Hospital, London, UK

2 ENT Specialist Registrar, ENT Department, Guy's Hospital, London, UK

3 Clinical Lead Speech and Language Therapist (Voice), Speech and Language Therapy Department, Guy's Hospital, London, UK

4 ENT and Laryngology Consultant, ENT Department, Guy's Hospital, London, UK of telephone or video consultations if possible. This was particularly advised for the most vulnerable patients [1]. Remote telephone triaging in head and neck cancer clinics has precedent during COVID-19 [2], utilising the validated head and neck cancer risk calculator [3-6]. There are many examples of clinical departments across the UK that have already incorporated and established telephone clinics into their practice, particularly for follow-up consultations [7-13]. In the age of digital technology, new platforms such as Babylon Health-GP at Hand have emerged and have started to increase in popularity [14]. However, the scale and necessity for a virtual style of consultation has never been greater than in the unprecedented circumstances of COVID-19.

ENT and specifically voice clinics carry a higher risk of disease transmission due to the frequent need for aerosolgenerating procedures (e.g. videolaryngostroboscopy) and necessitate the use of adequate personal protective equipment and FFP3 grade masks. Virtual clinics are able to 
capture relevant history, perceptual assessment of voice (GRBAS), quality of life measures (VHI-10), and potentially acoustic analysis to guide diagnosis and management [15-17]. The key drawback is that in the absence of endoscopic analysis it is not possible to confirm anatomical pathology and, therefore, difficult to progress patients to definitive treatment if surgery is anticipated.

In response to the onset of the coronavirus pandemic, from 23rd March our tertiary multi-disciplinary Laryngology-Speech Therapy voice clinic was converted to a wholly virtual laryngology specialist clinic, with consultations carried out via telephone or video. This mode of consultation continued exclusively until August, when following a sustained decline in cases, the clinic gradually switched back to fully face-to-face service, with additional evidence-based PPE measures in place. September and October 2020 have seen once more an increase in COVID-19 cases across many parts of Europe, including the UK [18] highlighting the need for a long-term flexible, responsive approach to working, which will likely involve a mixture of virtual, and face to face consultation methods.

\section{Aims}

The aims of our study were to

- Compare the effectiveness of virtual with face-to-face voice clinics in the assessment and progression of patient care.

- Understand patient satisfaction and perception of virtual voice clinics.

\section{Methods}

\section{Virtual clinic data analysis of clinic outcomes}

Virtual voice clinic data from clinics carried out over a 1-month period (28th April-25th May 2020) were reviewed and compared to data obtained from face-to-face clinics in a comparable time frame (1 November 2019-30 November 2019). Data were obtained from both electronic patient records and a dedicated voice clinic electronic database. All clinic letters were scrutinised, and relevant outcome data recorded; this included data on the type of clinic (new vs follow-up), primary complaint, diagnosis, and clinical outcome. Outcomes indicative of a clinically meaningful progression in patient care were identified and stratified into the following categories:

- Referred for imaging

- Referred to Speech and Language Therapy (SALT) and/ or another specialty

- Listed for surgery

- New medical therapy commenced

- Discharged

\section{Patient satisfaction survey}

All patients in the 1-month virtual clinic cohort were invited to complete a patient satisfaction survey (Table 1) and additionally provide unstructured feedback on their experience of virtual clinic. All patients were called by telephone, with a maximum of two attempts unless a scheduled call-back time was arranged. Data were collated and supplemented by a narrative review of patient feedback.

\section{Patient survey questions:}

1. Overall were you satisfied with how the virtual consultation went? Yes/No.

2. How long did the appointment last? Minutes.

3. Was the length of consultation satisfactory? Yes/No.

4. Was the telephone or video method of consultation satisfactory for addressing your health needs? Yes/No.

5. Would you have preferred to have waited until next possible physical review and examination? Yes/No.

6. Do you feel following this appointment that you would still benefit from physical review and examination? Yes/ No.

7. Would you like to have the option of telephone or video consultation in the future? Yes/No.

Table 1 Patient demographics

\begin{tabular}{|c|c|c|c|c|}
\hline & $\begin{array}{l}\text { Virtual clinic } \\
n=155\end{array}$ & & $\begin{array}{l}\text { Face-to-face clinic } \\
n=70\end{array}$ & \\
\hline Gender & $100(65 \%)$ Female & $55(35 \%)$ Male & $39(56 \%)$ Female & 31(44\%) Male \\
\hline Age & Range $17-86$ years old & & Range $22-90$ years old & \\
\hline Appointment & $76(49 \%)$ New & 79 (51\%) Follow-up & $46(66 \%)$ New & 24 (34\%) Follow-up \\
\hline Type of virtual call & 121 (78\%) Telephone & $\begin{array}{l}20(13 \%) \text { Video } \\
14(9 \%) \text { Unspecified/no letter }\end{array}$ & & \\
\hline
\end{tabular}

$n=155$ 


\section{Statistical analysis}

The ' $N-1$ ' Chi-square test of independence was performed to compare the proportions of progression in overall and outcome-specific patient care in virtual vs face-to-face groups, and patient feedback in new vs follow-up virtual patients, with $p<0.05$ considered statistically significant [19]. Calculations were completed using standard commercially available software (MedCalc v 19.6.1, Ostend, Belgium).

\section{Results}

Over a 1-month period (28th Apr to 25th May 2020), 155 patients were seen in 25 virtual clinics. Over a similar 1-month period pre-COVID-19 (1st to 30th Nov 2019), 70 patients were seen in 15 face-to-face clinics. Patient demographics are outlined in Table 1.

\section{Clinic outcomes}

Clinic outcome data are shown in Table 2. Overall a greater proportion of patients seen in face-to-face multi-disciplinary clinic had a clinically meaningful progression in their care
( $82 \%$ vs $55 \% p<0.0001)$. There was a statistically significant difference $(p<0.01)$ in the percentage of patients prescribed medical therapy ( $14 \%$ fewer), referred for SALT ( $21 \%$ fewer) or listed for surgery (13\% fewer) in the uni-disciplinary virtual clinic by comparison to the face-to-face, with only one patient listed for surgery. No statistical difference was found in the percentage of patients discharged from the clinic, or those referred for imaging or to other specialties.

\section{Patient satisfaction}

Results from the telephone patient survey are summarised in Table 3. We were able to speak to 75 patients. The feedback from the survey was overwhelmingly positive with $92 \%$ of patients saying that overall, they were satisfied with their virtual consultation. $98 \%$ of patients had prior communication in advance of their appointment. The median length of reported consultation was $15 \mathrm{~min}$, ranging from 5 to $40 \mathrm{~min}$ with $89 \%$ of patients feeling that the duration was adequate. The median time for those satisfied was $15 \mathrm{~min}$, those not satisfied was $10 \mathrm{mins}$ with an interquartile range of 15 and 10 , respectively. $60 \%$ of patients reported that the virtual method of consultation had been satisfactory in addressing their health needs; however, $84 \%$ thought they still would
Table 2 Patient outcomes: comparison of virtual vs. faceto-face clinics

Table 3 Patient survey result summary

\begin{tabular}{|c|c|c|c|c|c|c|}
\hline & \multicolumn{2}{|l|}{$\begin{array}{l}\text { Virtual clinic } \\
n=155\end{array}$} & \multicolumn{2}{|c|}{$\begin{array}{l}\text { Face-to-face clinic } \\
n=70\end{array}$} & \multirow[t]{2}{*}{$\%$ difference } & \multirow[t]{2}{*}{$p$ value } \\
\hline & No. of patients & $\%$ & No. of patients & $\%$ & & \\
\hline Discharges & 21 & & 614 & $20 \%$ & $6 \%$ & 0.255 \\
\hline Prescribed medication & 22 & & 20 & $28 \%$ & $14 \%$ & 0.012 \\
\hline Referred to SALT & 34 & & 630 & $43 \%$ & $21 \%$ & 0.001 \\
\hline Referred for imaging & 20 & & 68 & $11 \%$ & $2 \%$ & 0.674 \\
\hline Referral to another specialty & 10 & & $6 \quad 6$ & $8 \%$ & $2 \%$ & 0.577 \\
\hline Listed for operation & 1 & & 610 & $14 \%$ & $13 \%$ & 0.0001 \\
\hline $\begin{array}{l}\% \text { of patients where care was } \\
\text { progressed } \\
\text { *includes discharges }\end{array}$ & 86 & & & $82 \%$ & $27 \%$ & $<0.0001$ \\
\hline
\end{tabular}

\begin{tabular}{lll}
\hline & Number of patients/total & $\%$ \\
\hline Overall satisfaction & $69 / 75$ & $92 \%$ \\
Pre-clinic communication & $74 / 75$ & $98 \%$ \\
Average length of consultation & Median 15 min & \\
& Range 5-40 min & \\
Satisfactory length & Interquartile range 5 min & $89 \%$ \\
Satisfaction for meeting all health needs & $67 / 75$ & $60 \%$ \\
Prefer to wait till the next face to face & $45 / 75$ & $27 \%$ \\
Need to be seen face to face in future & $20 / 75$ & $84 \%$ \\
Would like the option of a virtual clinic in future & $63 / 75$ & $83 \%$ \\
\hline
\end{tabular}


benefit from a physical face-to-face review. $73 \%$ of patients said they would rather have had their virtual clinic than waited for a face-to-face review and $83 \%$ stated they would like the option of a telephone or video consultation in the future.

Despite a few technical issues, particularly with video methods, most patients had a smooth experience and reports of being 'pleasantly surprised' and 'impressed by the system' were commonplace. Groups who particularly highlighted the convenience of the service were those who lived far away from hospital, those with busy work or home schedules and those currently shielding due to COVID-19. Positive comments were received regarding the method 'saving lots of time' and being 'much more efficient and convenient'. Patients frequently commented that lack of physical examination was the main drawback of this type of clinic with comments such as 'you can't beat face to face' and 'I would have preferred in person' being characteristic. A few patients mentioned in the discussion that little was achieved without a physical examination and that it resulted in duplication of appointments.

A significant proportion of patients were particularly positive about virtual speech and language therapy sessions that they had started as a result of virtual voice clinic referrals. The overriding feedback was that virtual methods are acceptable in the right context but not an appropriate wholesale substitute for face-to-face consultation. Whilst opinion varied amongst patients, a majority expressed that follow-up virtual appointments would be more appropriate.

Patients who expressed particular difficulty with virtual voice clinic were those whose voice issues were so severe that they were unable to easily converse over the phone, those with cognitive difficulties or autism or language barriers for whom communication was more challenging.

Statistical analysis was made to evaluate whether clinic type (new consultation vs follow-up) affected patient feedback and satisfaction. Table 4 and Graph 1 outline the results. Chi-squared analysis of independence was calculated and showed there was no statistical difference between any of the parameters of the questionnaire.

\section{Discussion}

The COVID-19 pandemic has prompted us to adapt to new methods of working and its unpredictable course means we will need to work flexibly with a combination of virtual and face-to-face clinics as circumstances demand. We need to evaluate whether positive elements of virtual working can be taken forward into standard practice in the longer term. With this in mind, the main questions are (a) how effective are virtual clinics vs face-to-face clinics in progressing patients'
Table 4+Graph Virtual clinic patient satisfaction according to clinic type

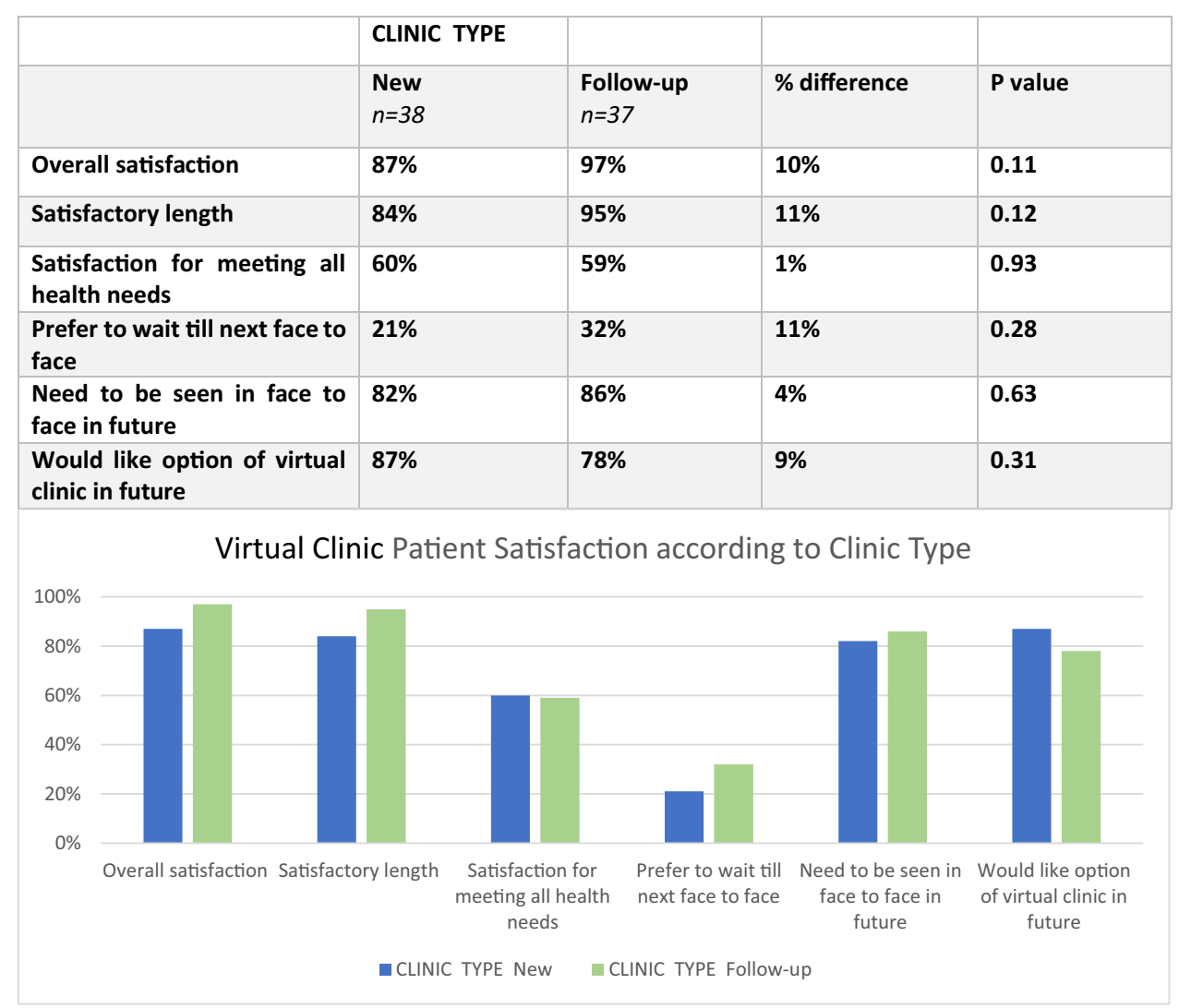


care in combined Laryngology-Speech Therapy voice clinics and (b) what is patient satisfaction with virtual methods of consultation.

\section{Progressing patient care and patient feedback}

Our outcome data demonstrate that there is a large statistically significant difference in the proportion of patients whose care progressed, indicating that face-to-face clinics remain preferable. In spite of this, care was progressed in a clinically meaningful way in over $50 \%$ of virtually reviewed patients, demonstrating its value as an alternative when necessary. This was mirrored in high satisfaction rates. Unsurprisingly, due to the lack of clinical examination to confirm pathology, only $1(<1 \%)$ patient from the virtual clinic cohort was listed for surgery, this was a general ENT patient mistakenly triaged into voice clinic. She was listed for a tonsillectomy due to recurrent tonsilitis. History and video-link oral examination were sufficient in this case; however, for new truly voice patients, the number of patients listed would undoubtedly have been $0 \%$. Clinicians found that without laryngoscopy, initial diagnosis and assessment of progress was hindered and that this crucial limitation impeded management in most patients, particularly regarding surgical planning. The context for which laryngoscopy was occasionally deemed unnecessary was in some followup patients established on treatment where patient-reported symptoms were sufficient. However, clinicians were fully satisfied with this alone in few cases.

Over double the number of patients were seen virtually (155 vs 70) in a similar timeframe. This was attributable to 10 more clinics taking place over the lockdown month (in place of cancelled theatre sessions), and non-inclusion of specific patients (i.e. vocal cord checks/non-voice patients/ non-attendances) in the face-to-face database cohort rather than virtual clinics being doubly 'efficient'. That said, with shorter average appointment times and as our familiarity with virtual platforms improves, the capacity for patients on virtual clinics may be slightly greater.

A number of factors limit our conclusions at present. Whilst patient satisfaction appears high, the absence of feedback from the face-to-face group precludes direct comparison, and prospective comparison of intergroup patient satisfaction is warranted in future studies to obtain further insights into this. Second, our two points of data collection took place in winter and spring respectively, where marked differences in rates of viral and allergy-related illness may have impacted patient management. Finally, it is difficult to extrapolate as to the durability of the high levels of patient satisfaction, which maybe have been charitable and context specific, and indeed may fatigue as we enter subsequent waves of pandemic-related lockdown measures.
Going forward there may be benefits of combined virtual Laryngology-Speech Therapy voice clinics for specific patients outside of the COVID-19 circumstances. This includes patients travelling from far away, those struggling to take time off work or away from caring responsibilities, and those under active surveillance. It may also be beneficial to triage new patients before booking into face-to-face clinics, with our data suggesting that some patients can be discharged without face-to-face appointments, although this would undoubtedly result in duplication of many appointments. In the future, an ideal clinic may be a hybrid of faceto-face and virtual appointments as clinically indicated. This could have cost-saving implications, reduce waiting lists and improve clinic attendance if virtual appointments are generally quicker with greater capacity in clinics. Awareness that virtual methods do not suit all patients is important and an element of patient choice is essential.

Additionally, virtual platforms of working create a potential for clinicians to work from home with remote access. In a society where work-life balance and flexibility to work from home are rapidly becoming a priority for all sectors, this may become a reality for medical and surgical departments where such opportunities have previously seemed unfeasible.

Availability of data and material Data are displayed in the figures of this article. Access to anonymised databases can be given on request.

\section{Compliance with ethical standards}

Conflict of interest No known conflict of interests.

\section{References}

1. ENT UK (2020) Guidance for ENT during the COVID-19 pandemic. https://www.entuk.org/guidance-ent-during-covid-19pandemic. Accessed September 2020

2. ENT UK (2020) Remote triaging or urgent suspected head and neck cancer referrals during COVID-19 pandemic. https://www. entuk.org/sites/default/files/files/ENTUK_2WW_Telephone_Triage_Letter.pdf. Accessed September 2020

3. Tikka T, Kavanagh K, Lowit A et al. (2020) Head and neck cancer risk calculator (HaNA-RC) - V.2. Adjustments and addition of symptoms and social history factors. Clin Otololaryngol 45(3):380-388) https://doi.org/10.1111/coa.13511.

4. Tikka T, Paleri V, MacKenzie K (2018) External validation of a cancer risk prediction model for suspected head and neck cancer referrals. Clin Otolaryngol 43(2):714-717. https://doi.org/10. 1111/coa.13019

5. Tikka T, Pracy P, Paleri V (2016) Refining the head and neck cancer referral guidelines: a two-centre analysis of 4715 referrals. Clin Otolaryngol 41(1):66-75) https://doi.org/10.1111/coa.12597.

6. HANC-RC v.2: Symptom Based Risk Calculator for Head and Neck Cancer Referrals v2 (2019) http://www.orlhealth.com/riskcalculator-2.html. Accessed September 2020 
7. Healy P, McCrone L, Tully R et al (2018) Virtual outpatient clinic as an alternative to an actual clinic visit after surgical discharge: a randomised controlled trial. BMJ Quality Safety 28:7-9. https:// doi.org/10.1136/bmjqs-2018-008697

8. Rosbe KW, Jones D, Jalisi S et al (2000) Efficacy of postoperative follow-up telephone calls for patients who underwent adenotonsillectomy. Arch Otolaryngol Head Neck Surg 125(6):718-21 https://doi.org/10.1001/archotol.126.6.718

9. McVay MR, Kelley K, Mathews D et al (2008) Postoperative follow-up: is a phone call enough? J Pediatr Surg 43(1):83-86. https://doi.org/10.1016/j.jpedsurg.2007.09

10. Gray RT, Sut MK, Badger S et al (2010) Post-operative telephone review is cost-effective and acceptable to patients. Ulster Med J 79(2):76-79

11. Fallaize R, Tinline-Purvis C, Dixon A, Pullyblank A (2008) Telephone follow-up following office anorectal surgery. Ann R Coll Surg Engl 90(6):464-466. https://doi.org/10.1308/003588408X 300975

12. Ryan E, Hanly A, McCawley N et al (2013) A virtual outpatient department provides a satisfactory patient experience following endoscopy. Int J Colorectal Dis 29:359-364. https://doi.org/10. 1007/s00384-013-1801-y

13. Thompson-Coon J, Abdul-Rahman A, Whear R et al (2013) Telephone consultations in place of face to face out-patient consultations for patient discharged from hospital following surgery: a systematic review. BMC Health Serv Res. https://doi.org/10.1186/ 1472-6963-13-128

14. Babylon: GP at Hand https://gpathand.nhs.uk Accessed September 2020

15. Sáenz-Lechón N, Godino-Llorente J, Osma-Ruiz V et al (2006) Automatic assessment of voice quality according to the GRBAS scale. Con Proc IEEE ENg Med Biol Soc 2006:2478-2481. https://doi.org/10.1109/IEMBS.2006.260603

16. Rosen C, Lee A, Osborne J et al (2004) Development and validation of the voice handicap index -10. Laryngoscope 114(9):15491556. https://doi.org/10.1097/00005537-200409000-00009

17. Scott-Brown WG, Watkinson JC, Clarke RW (2018) Evaluation of the Voice. Scott-Brown's Otorhinolaryngology and Head and Neck Surgery, 8th edition. CRC Press, Chapter

18. Gov.UK (2020) Coronavirus (COVID-19) in the UK Dashboard. https://coronavirus.data.gov.uk/ Accessed October 2020

19. Campbell I (2007) Chi-squared and Fisher-Irwin tests of twoby-two tables with small sample recommendations. Stat Med 26:3661-3675

Publisher's Note Springer Nature remains neutral with regard to jurisdictional claims in published maps and institutional affiliations. 